\title{
FURTHER APPLICATIONS OF THE PHOTOGRAPHIC METHOD IN NUCLEAR PHYSICS
}

\author{
By DR. C. F. POWell, \\ The W'ills Physical Laboratory, University of Bristol
}

$\mathrm{I}^{\mathrm{N}}$ a letter published in Nature of July 15, 1939, p. 115, attention was directed to the fact that the photographic plate provides a very powerful instrument for investigations depending on the detection and measurement of the energy of heavy charged particles, notably protons, and of neutrons. It was shown that the method, when applied to determining the energies of the neutrons liberated from the light elements under deuteron bombardment, yields essentially the same information as that

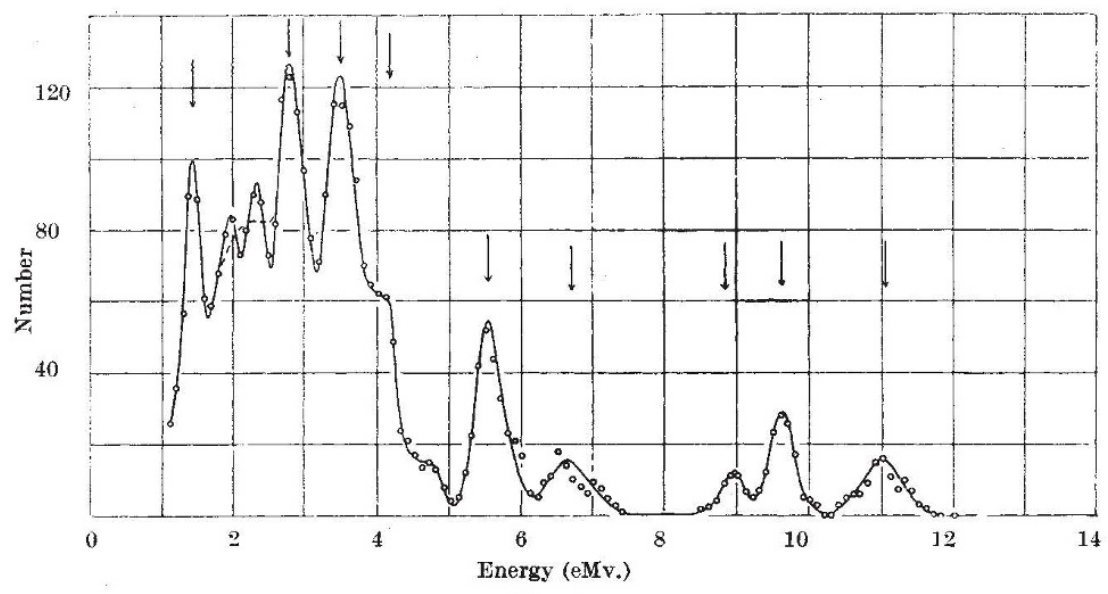

Fig. 1,

FORWARD NEUTRONS FROM $\mathrm{F}+\mathrm{D}$ AT $900 \mathrm{KV}$

Number per $200 \mathrm{kv}$. range plotted for every $100 \mathrm{kv}$. Peaks statistically justified are shown by arrows. Two tracks were also observed, at 13.2 and $13.6 \mathrm{eMv}$. respectively, which suggest slight boron contamination of the target.

ments of the tracks were made by a single observer in about sixty working hours. We understand that the same spectrum has been investigated by $\mathrm{Dr}$. T. W. Bonner using the expansion chamber, and that his results will be published shortly. We have not seen his results, and it will thus be possible to make a completely independent check on the validity of the photographic method. Two tracks of $13.4 \mathrm{eMv}$. protons suggest boron contamination of the target, and this may be responsible for the peaks at $4 \cdot 3$ and $9 \cdot 0 \mathrm{eMv}$. Further work will be necessary to decide this point.

It was suggested in the previous letter that the photographic method is equivalent to a continuously sensitive expansion chamber. We believe it possible to press the analogy further in the sense that the spatial orientation of a track in the emulsion can be determined. With objectives of high aperture, the depth of focus is so small that it is possible to determine the depth of individual grains in the emulsion with a high degree of precision, and hence the angle of inclination of a track to the plane of the plate. In addition, the direction of the projection of the track on the plane of the plate can be measured to within a quarter of a degree

provided by the expansion chamber. The photographic method has, however, marked advantages over the expansion chamber, which were described.

The first experiments were made on neutrons of which the energy spectrum had already been investigated. It was, therefore, of importance to apply the method to an investigation in which previous work could not serve as a guide able to prejudice the experimental observations. Adequate checks were applied in the previous work and it is believed that the results are free from this objection, but nevertheless an independent experiment was desirable. Through the courtesy of Prof. J. D. Cockeroft and Mr. P. I. Dee, it was possible to examine the neutrons from fluorine under bombardment with $900 \mathrm{kv}$. deuterons from the Cambridge one million-volt generator. The results are shown in Fig. 1, and are taken from measurements on 1,700 tracks contained in $4 \mathrm{sq}$. cm. of an Ilford half-tone plate of which the emulsion was $100 \mu$ thick. The results were obtained with an exposure of two hours with 30 microamperes in the resolved deuteron beam, the plate being at a distance of about $10 \mathrm{~cm}$. from the target and in a position to detect forward neutrons. The measure- and the direction in space thus defined. We have examined the distribution in angle of the protons ejected by the neutrons from boron under deuteron bombardment as they pass though the plate.

We and other observers have shown that the highenergy neutrons from this reaction fall into four groups with energies of approximately $4,6,8 \cdot 7$ and $13 \cdot 3$ eMv. when the bombarding deuterons have an energy of $600 \mathrm{ekv}$. If a neutron of energy $\boldsymbol{E}_{0}$ is scattered by a proton, the energy acquired by the proton, if projected at an angle $\beta$ with the direction of the incident neutrons, is $E_{0} \cos ^{2} \beta$. If we measure the range of protons projected in a photographic plate by the boron neutrons, we should therefore expect the ranges of the observed tracks to be distributed, according to the angle $\beta$, in a way corresponding to this energy equation. We should expect the points to lie, not on four lines, but within four bands as a consequence of :

(a) the straggling of the protons,

(b) the uncertainty of $\pm 2^{\circ}$ in the direction of the neutrons entering the plate, owing to the width of the deuteron beam striking the target, and

(c) an uncertainty of $\pm 1^{\circ}$ in the determination of $\beta$. 


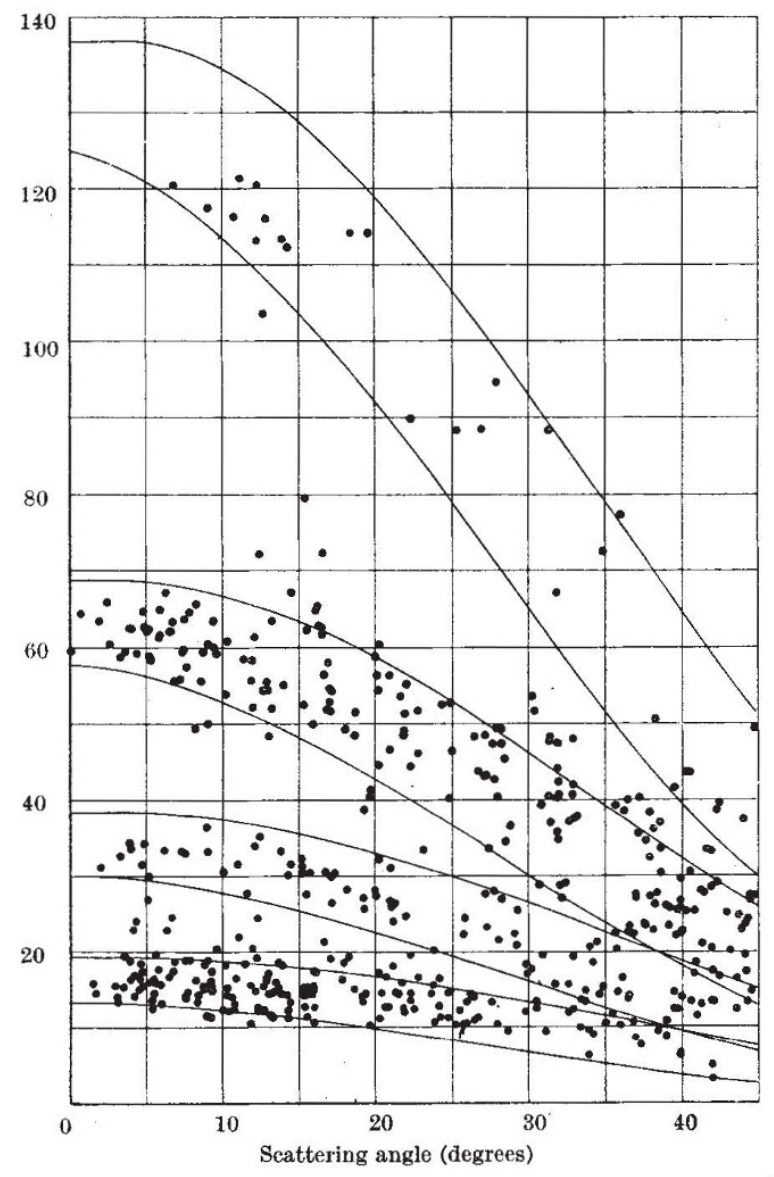

Fig. 2.

DISTRIBUTION OF RANGE WITH ANGLE FOR 500 PROTON TRACKS SCATTERED BY B + D NETTRONS.

Tracks measured make angles up to $\pm 12^{\circ}$ with plane of the plate. Range is plotted in microscope eyepiece scale divisions.

The bands shown in Fig. 2 are drawn by assuming a 'width' of $0.8 \mathrm{eMv}$. in the range at any angle due to straggling, and an uncertainty of $\pm 3^{\circ}$ in the determination of $\beta$ due to factors $(b)$ and $(c)$. The results for the first 500 tracks measured are shown as points in Fig. 2, and it will be seen that 90 per cent of the tracks lie within the four bands. The results emphasize in a striking way one of the conclusions of the previous letter, that the photographic method has the advantage over the expansion chamber in that the amount of matter in the plate is so small that it does not give rise to scattered neutrons in serious numbers, and show that it is possible to investigate neutron-proton scattering at high energies by the photographic method. The measurements are proceeding.

The possibility of determining $\gamma$-ray energies by means of the photographic plate has also been investigated. Plates loaded with heavy hydrogen were exposed to the $\gamma$-rays from fluorine under proton bombardment and a search made for the photo-disintegration protons. The difficulty is that the cross-section per atom for the production of electrons in the emulsion by the $\gamma$-rays is about a thousand times greater than that for the photodisintegration of the deuteron. Although many electrons must pass through a grain before it will develop, a limit is thus imposed on the time of exposure to which the plate can be subjected, beyond which the general background of fog makes it impossible to distinguish proton tracks. With the first plates available the deuterium content was too small to give a sufficient number of tracks per sq. $\mathrm{cm}$. of the plate at the limiting $\gamma$-ray exposure. A layer of 'heavy' ammonium chloride, $\left(\mathrm{ND}_{4}\right) \mathrm{Cl}$, was therefore supported immediately above an ordinary half-tone plate during an exposure to the $\gamma$-rays. A large number of proton tracks was then observed in the developed plate, starting in the surface of the emulsion, and having a maximum energy of $2 \mathrm{eMv}$. We attribute these to the photo-disintegration of the deuterium under the action of the $6 \mathrm{eMv} . \gamma$-rays from the fluorine.

Although the application of the method to the determination of $\gamma$-ray energies ean only be made in the absence of neutrons, it can be used in its present form to determine the spatial distribution of protons from the photo-disintegration process, an experiment which is of great importance for the meson field theory. This experiment, and further work with plates loaded with greater amounts of heavy hydrogen, is proceeding.

It is evident from the results published in our previous communication on the protons from the reaction $\mathrm{B}^{10}-d, p$ that the photographic method can be applied to scattering experiments between fundamental particles, $p-p, p-d, p-\alpha$, etc., using the high-energy protons provided by the cyclotron, and we are at present collaborating with Prof. J. Chadwick on such experiments. Dr. T. R. Wilkins kindly informs me in a private communication that he is investigating proton-proton scattering at high energies by a similar method. The great advantage of the method for such work is that, in contrast with experimental methods employing counters, the particles scattered through different angles are all recorded at the same time, so that it is not necessary to apply elaborate corrections for maintaining the constancy of the bombarding stream or correcting for its variation. The analysis of the plates is much easier in these experiments than with neutron work, since the scattered particles all start in the surface and run parallel into the plate. Dr. Wilkins employs a camera carrying a large number of plates in this

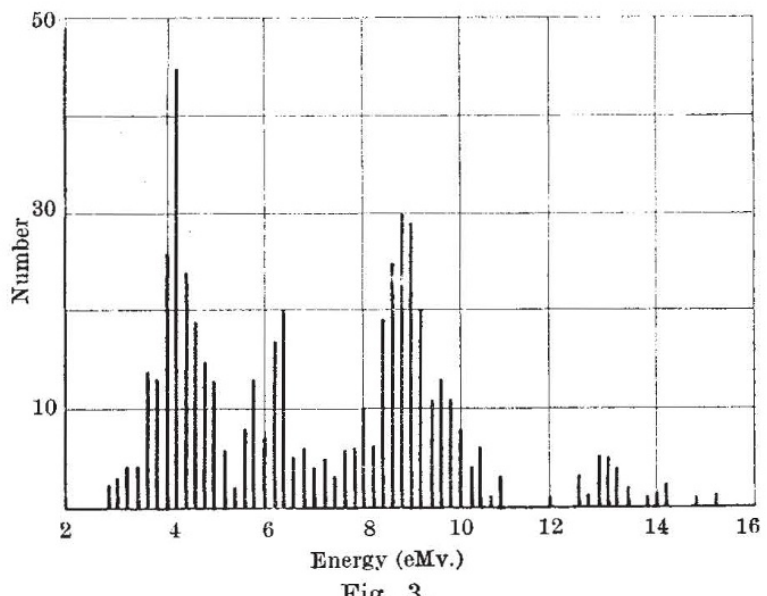

Fig. 3.

ENERGY DISTRIBUTION FROM ALL MEASURED TRACKS PROJECTED WITHIN $40^{\circ}$ OF THE DIRECTION OF THE INCIDENT NEUTRONS. 
type of experiment. We use a single rectangular plate with the proton beam running parallel to the long axis and a little above the plate. The beam, defined by stops, passes down the axis of a tube provided with a short interruption through which protons seattered by the gas in the camera can emerge to enter the plate.

A detailed account of the methods employed will shortly be submitted for publication in another place. It may be mentioned here that we find it a considerable advantage to use binocular microscopes and optical equipment of the highest quality to reduce the nervous strain on the observer to a minimum. Although the Ilford half-tone plate has proved very adequate for the work, it seems likely that it will be possible to develop special plates with superior characteristics for nuclear work.

\section{THE VOCODER}

\begin{abstract}
$\mathbf{A}^{\mathrm{T}}$ the World's Fairs in New York and San A Franciseo great interest was shown in the speech synthesizer shown in the Bell System exhibits. In the December number of the Bell Laboratories Record, H. Dudley, of their research department, describes this device, known as the 'vocoder'. The 'voder' is an offshoot of a more extensive system first demonstrated in its experimental stage several years ago. It first analysed spoken sounds and then used the information to control the synthesizing eircuit. As World's Fair displays were then under consideration it was seen that the synthesizer, manually controlled, could be made into a dramatic demonstration. Development was at first concentrated in this field, but when a successful Voder became assured, attention was shifted back to the broader and parent system and it was called the 'vocoder', since it operates on the principle of deriving voice codes to re-create the speech which it analyses. The analyser is at the left and the synthesizer at the right of the vocoder.
\end{abstract}

Electrical speech waves from a microphone are analysed for pitch by one channel and for spectrum by a group of channels. In the pitch analysis the fundamental frequency is called for simplicity the pitch. It is measured by a circuit containing a frequency discriminating network which obtains the frequency in a pure form, a frequency meter for counting, by more or less uniform pulses, the current reversals therein, and a filter for eliminating the actual speech frequencies but retaining a slowly changing current that is a direct measure of the pitch. The output current of the pitch channel is then a pitch-defining channel with its current approximately proportional to the pitch of the voiced sound and equal to zero for the unvoiced sounds.

There are ten spectrum-analysing channels, the first handling the frequency range 0-250 cycles and the other nine, the bands, 300 eycles wide, extending from 250 cycles to 2,950 cycles, a top frequency which is representative of commercial telephone eircuits. The operation of the analyser is illustrated by a group of oscillograms taken in analysing the sentence "She saw Mary". In the analyser the speech wave is the input and the eleven speechdefining signals are the output; in the synthesizer the eleven speech-defining signals are the imput and the speech wave the output. The relaxation oscillator is the source of the buzz, and the random noise circuit the source of the hiss. When a voiced sound is analysed a pitch current other than zero is received, with the result that the buzz is set for the current pitch by the "pitch control" on the relaxation oscillator; also, the relay marked "energy source switch" operates, switching from the hiss source to the buzz source. From the loud-speaker comes speech approximately the same in pitch and in spectrum as the original.

The possibilities of the Vocoder were recently demonstrated by the author before the Acoustical Society of America and before the New York Electrical Society. In these demonstrations comparison is first made between direct speech and the best recreation that the apparatus could make. Then by manipulation of dials and switches, speech is modified in various ways. Normal speech becomes a throaty whisper when the hiss is substituted for the buzz. Although the hiss is relatively faint, it is shown to be essential for discrimination between words like 'church' and 'shirts'.

Ordinarily the re-created pitch moves up and down with that of the original. If variation is prevented, the re-created speech is a monotone like a chant. When the relative variation is cut in half, the voice seems flat and dragging; when the swings are twice normal, the voice seems more brilliant; when four times normal it sounds febrile and unnatural. The controls can be reversed, so that high becomes low ; the tune of a song is then unrecognizable, and speech has some of the lilting characteristics of Scandinavian tongues. By appropriate setting of the basic pitch, the voice may be anything from a low bass to a high soprano. If the basic pitch is set to maintain a constant ratio of, say, 5 to 4 to the original, this is a 'major third' higher and harmonizes with the original. In two-part harmony, the demonstrator can then sing a duet with himself. Connecting a spare synthesizer for a 3 to 4 ratio he can sing one part in a trio, the others being taken by his electrical doubles. Finally, with the basic pitch control of the apparatus, he becomes a father reprimanding his daughter, then the girl herself, and then becomes the grandfather interceding for the child. Keeping careful time with the puffs of a locomotive, the demonstrator can make the locomotive puff intelligibly "We're--starting - slowly-faster, faster, faster" as the puffs come closer together. A particularly striking effect is that of singing with an organ to supply the tones. Although the words may be spoken, the demonstrator usually sings them to hold the rhythm. It makes no difference whether his voice is melodious or not; the tonal quality comes only from the musical source.

The engineering possibilities which may grow out of the application of the principles employed in this device are hard to predict at the present time. The speech-defining currents, however, do have features of simplicity and inaudibility which may open the way to new types of privacy systems or to a reduction in the range required for the transmission of intelligible telephonic speech. 